\title{
The Impact of Conscious Sedation versus General Anesthesia for Stroke Thrombectomy on the Predictive Value of Collateral Status: A Post Hoc Analysis of the SIESTA Trial
}

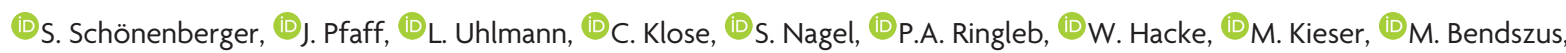 \\ (1) M.A. Möhlenbruch, and ${ }^{-}$J. Bösel
}

因正

\begin{abstract}
BACKGROUND AND PURPOSE: Radiologic selection criteria to identify patients likely to benefit from endovascular stroke treatment are still controversial. In this post hoc analysis of the recent randomized Sedation versus Intubation for Endovascular Stroke TreAtment (SIESTA) trial, we aimed to investigate the impact of sedation mode (conscious sedation versus general anesthesia) on the predictive value of collateral status.

MATERIALS AND METHODS: Using imaging data from SIESTA, we assessed collateral status with the collateral score of Tan et al and graded it from absent to good collaterals $(0-3)$. We examined the association of collateral status with 24-hour improvement of the NIHSS score, infarct volume, and $\mathrm{mRS}$ at 3 months according to the sedation regimen.

RESULTS: In a cohort of 104 patients, the NIHSS score improved significantly in patients with moderate or good collaterals (2-3) compared with patients with no or poor collaterals $(0-1)(P=.011$; mean, $-5.8 \pm 7.6$ versus $-1.1 \pm 10.7)$. Tan $2-3$ was also associated with significantly higher ASPECTS before endovascular stroke treatment (median, 9 versus $7 ; P<.001$ ) and smaller mean infarct size after endovascular stroke treatment (median, 35.0 versus 107.4; $P<.001$ ). When we differentiated the population according to collateral status $(0.1$ versus 2.3 ), the sedation modes conscious sedation and general anesthesia were not associated with significant differences in the predictive value of collateral status regarding infarction size or functional outcome.
\end{abstract}

CONCLUSIONS: The sedation mode, conscious sedation or general anesthesia, did not influence the predictive value of collaterals in patients with large-vessel occlusion anterior circulation stroke undergoing thrombectomy in the SIESTA trial.

ABBREVIATIONS: $\mathrm{EST}$ = endovascular stroke treatment; $\mathrm{CS}$ = conscious sedation; $\mathrm{GA}$ = general anesthesia; $\mathrm{RCT}$ = randomized trial; SIESTA = Sedation versus Intubation for Endovascular Stroke TreAtment; Tan = collateral score of Tan et al ${ }^{16}$

$\mathbf{E}^{\mathrm{n}}$ ndovascular stroke treatment (EST) is now the first choice for acute ischemic stroke in the anterior circulation caused by large-vessel occlusion. ${ }^{1,2}$ However, selection criteria to identify patients likely to benefit from EST outside highly selective randomized trials (RCTs) are still controversial. It is also important to establish practicable selection criteria for thrombectomy failure to exclude patients prone to futility and save financial, facility,

Received March 2, 2017; accepted March 17

From the Departments of Neurology (S.S., S.N., P.A.R., W.H., J.B.) and Neuroradiology (J.P., M.B., M.A.M.), Heidelberg University Hospital, Heidelberg, Germany; and Institute of Medical Biometry and Informatics (L.U., C.K., M.K.), University of Heidelberg, Heidelberg, Germany.

S. Schönenberger and J. Pfaff contributed equally to this work.

The study was performed with departmental funding only.

Please address correspondence to Silvia Schönenberger, MD, Im Neuenheimer Feld 400, 69120 Heidelberg, Germany; e-mail: silvia.schoenenberger@med.uni-

heidelberg.de

三 Indicates article with supplemental on-line tables.

http://dx.doi.org/10.3174/ajnr.A5243 and personnel resources and, above all, avoid complications like cerebral reperfusion injuries.

Current data suggest that collateral blood flow status is a strong independent predictor of therapeutic success and functional outcome after EST. ${ }^{3-5}$ By a network of pre-existing anastomoses, compensatory cerebral collateral blood flow supplies oxygen-deprived brain areas to which the primary flow path is blocked due to large-vessel occlusion. The recently published post hoc analysis on the collateralization status from the seminal thrombectomy trial MR CLEAN (Multicenter Randomized Clinical Trial of Endovascular Treatment of Acute Ischemic Stroke in the Netherlands) showed the highest interventional therapeutic effect in patients with moderate-to-good collateral blood flow. ${ }^{6}$

Why should the chosen sedation/airway regimen influence the impact of collateralization during EST? Collateral effects depend on cerebral perfusion pressure and vasomotor regulation of the vessel diameter. On the one hand, intubation and general anesthesia (GA) are often associated with a substantial drop in blood pressure $^{7}$ as was shown in previous EST studies. ${ }^{8-10}$ It is quite 
likely that hypotension may compromise even patients with a good collateral status, particularly if their cerebral autoregulation is impaired as is often the case in severe acute ischemic stroke. Moreover, inadvertent mechanical hyperventilation and subsequent hypocarbia can lead to cerebral vasoconstriction. ${ }^{8,11}$ Both hypotension and hypocarbia associated with GA may have disadvantageous effects on the insufficiently supplied penumbra. Indeed, many retrospective studies have suggested worse outcome and mortality associated with GA in EST. ${ }^{12}$ On the other hand, steering GA in ways that stabilize circulation and aim for normocarbia may theoretically serve to improve collateralization.

We recently conducted the Sedation versus Intubation for Endovascular Stroke TreAtment (SIESTA) study to compare GA with conscious sedation (CS) during EST. ${ }^{13}$ In that first RCT on peri-interventional management, strict target values for physiologic parameters, including blood pressure and $\mathrm{CO}_{2}$, were predefined for both treatment groups, mainly to avoid hypocarbia and hypotension. SIESTA showed no difference between GA and CS with regard to early neurologic improvement measured by the NIHSS after 24 hours, ${ }^{14}$ and unadjusted long-term outcome was even better in patients in the GA group. Of note, SIESTA was not powered and designed to primarily investigate long-term functional outcome, and slight imbalances in reperfusion grades, for example, may still have influenced that result even though the results were not statistically significant. This result was in strong contrast to most previous, yet retrospective, studies on the subject.

Why was GA not inferior in SIESTA? We hypothesized that the protocolized way GA was conducted may not have compromised or even improved collateralization. In this post hoc analysis of imaging data from the SIESTA study, we mainly aimed to investigate whether the predictive value of collateral status for infarct volume and outcome is affected by the applied sedation mode (CS versus GA) and, more specifically, whether patients under a very standardized GA may have shown a favorable course despite a suboptimal collateral status at baseline.

\section{MATERIALS AND METHODS Design of the SIESTA Trial}

Patient eligibility and the methods, including the study protocol, were previously published. ${ }^{13}$

Briefly, SIESTA was a monocentric, prospective, randomized, parallel-group, open-label treatment trial with blinded end point evaluation. Patients with an NIHSS score of $>10$ and isolated or combined occlusion of the internal carotid artery or middle cerebral artery selected for EST were randomized 1:1 to either the nonintubated state in CS or to the intubated state in GA. The trial was approved by our institutional review board (Ethikkommission Medizinische Fakultät Heidelberg, ID S-650/2013).

\section{Main Outcomes of the SIESTA Trial}

The main finding of SIESTA was no superiority of CS versus GA with regard to early neurologic improvement (change in the NIHSS score from admission to 24 hours thereafter). ${ }^{14}$ There were no differences in most secondary outcomes between the groups either (including in-hospital and 3-month mortality, and variables of peri-interventional safety and feasibility).
Secondary radiologic outcomes relevant to the present analysis included the ASPECTS, ${ }^{15}$ the collateral status based on CTA on admission described by Tan et $\mathrm{al}^{16}{ }^{16}$ the modified $\mathrm{TICI}^{17}$ score based on DSA at the end of EST, and the final infarct volume on follow-up CT at 18-24 hours after EST. ${ }^{18}$ Only patients with a full dataset (baseline CT including CTA, ASPECTS before EST, and follow-up CT within 18-24 hours after onset) were included in this post hoc analysis. More details and definitions can be found in the earlier publication of the SIESTA protocol. ${ }^{13}$

\section{Imaging Data Assessment and Analysis}

All CT and CTA data were evaluated by 2 independent, experienced neuroradiologists who were blinded to clinical findings, type of anesthesia, and infarction side (J.P., M.A.M.). The ASPECTS score, which ranges from 0 to 10 , was used as a quantitative measure of areas with early infarction signs, with higher scores indicating fewer ischemic changes. ${ }^{15}$

Collaterals of the CTA source images were evaluated with the collateral score of Tan et $\mathrm{al}^{16}$ and divided into the 4 categories: 0 (no collaterals; no filling of the occluded area), 1 (poor collaterals; $>0 \%$ but $<50 \%$ filling of the occluded area), 2 (moderate collaterals; $>50 \%$ but $<100 \%$ filling of the occluded area), and 3 (good collaterals; $100 \%$ filling of the occluded area), yielding the 2 groups with scores of $0-1$ and 2-3. CTA was mostly performed in combination with noncontrast CT as part of our in-house protocol for stroke imaging but was sometimes repeated in patients transferred from other hospitals after external CTA. The latter was then used as a baseline for EST. Patients without a baseline CTA and ASPECTS were excluded from this analysis.

\section{Statistical Analysis}

For continuous data, mean $( \pm S D)$ and median (interquartile range) values are presented. Absolute and relative frequencies are given for categoric and ordinal variables. In a first explorative analysis, patients were divided into 2 Tan groups: good-to-moderate versus poor collaterals ( $0-1$ versus $2-3)$. To test for differences between the groups, we applied $t$ tests, $\chi^{2}$ tests, and MannWhitney $U$ tests, as appropriate. In a second step, we divided the 2 groups into subgroups according to treatment arm in the SIESTA trial (CS or GA). Again, a descriptive analysis and tests were applied to look for any differences within the subgroups. In a third step, we applied linear and logistic regression analyses to examine the predictive value of Tan, ASPECTS, and the randomization arm on the NIHSS score after 24 hours; the mRS after 3 months; and final infarct volume. In addition, we added an interaction term between Tan and the randomization variable that was only included in the final model if it showed a significant influence on the outcome value. In a final step, Pearson or Spearman correlations of improvement of NIHSS, Tan, final infarct volume, and ASPECTS were estimated. We also conducted post hoc power analyses.

A $P$ value $<.05$ was considered statistically significant. Because this was an explorative study, no adjustment for multiple testing was performed. For statistical analyses, we used SAS software (SAS Institute, Cary, North Carolina), Version 9.4, and R, Version 3.3.1. ${ }^{19}$ 


\section{RESULTS}

\section{Patient Characteristics}

For the present analysis, 104 of 150 patients in SIESTA fulfilled the study-specific inclusion criteria. Of the 46 excluded patients, 43 had not received a baseline CTA before EST, and in 3, the final infarct volume after EST could not be determined due to symptomatic hemorrhage. The group with Tan 2-3 had a significantly better ASPECTS $(P<.001)$; 83.9\% had an ASPECTS of 8-10 compared with only $50 \%$ with Tan $0-1$. The group with Tan $2-3$ had a significantly poorer pre-mRS $(P=.02)$ compared with Tan $0-1$. Eight patients had to be converted from CS to GA (5 Tan $0-1$ and 3 Tan 2-3). Further baseline, demographic, and clinical characteristics of the 2 collateral groups are shown in Table 1.

\section{Outcomes According to Collateral Status}

Tan $0-1$ was associated with a significantly higher mean final infarct size ( 107.4 versus $35.0 \mathrm{~cm}^{3} ; P<.001$ ) compared with Tan $2-3$. A statistically significant improvement in the mean NIHSS score after 24 hours was found in patients with Tan 2-3 (mean, $-5.8 \pm 7.6$ versus $-1.1 \pm 10.7 ; P=.01$ ). Functional independence after 3 months measured by mRS $0-2$ was not significantly different. Significantly lower in-house mortality $(14.6 \%$ versus $1.8 \%, P=.02$ ), but no higher 3 -month mortality (26.8\% versus $25 \%, P=.84$ ) was found in Tan 2-3. Finally, the mean length of stay of patients with Tan 2-3 in the neuro-intensive care unit was significantly shorter (mean values, 2.7 versus 8.2 days; $P<.001$ ). The post hoc power analysis comparing Tan $0-1$ versus 2-3 showed a power of $73.4 \%$ for the improvement of the NIHSS score after 24 hours, $99.6 \%$ for infarct size, and 68.5\% for inhouse mortality. There were no significant differences in the analysis of short-term peri-interventional safety and feasibility parameters between the collateral groups (Table 2).

\section{Impact of Collateralization According to Sedation Mode}

When the 2 collateralization groups were further subdivided according to the applied sedation mode used in SIESTA (CS versus GA), the only baseline difference was that patients with Tan $0-1$ treated with GA were younger (mean, 75.5 versus 68.8 years; $P<$ .005). Most outcomes were not different in comparison.

In the subgroup with Tan $0-1$, the only significant differences were more long-term functional independence (mRS after 3 months dichotomized to $0-2$ and $3-6$, CS $8.7 \%$ versus GA $32 \%$; $P=.05)$ and more delayed extubations (17.4\% versus 52\%; $P=$ $.01)$ in GA.

In the group with Tan 2-3, the GA subgroup was associated with a significantly longer mean time to treatment (104.3 versus 152.0 minutes; $P=.01)$, higher ASPECTS $(P=.05)$, a higher rate of hypothermia $(10.3 \%$ versus $33 \%, P=.04)$, and a higher rate of delayed extubation $(3.4 \%$ versus $40.7 \%, P<.001)$. The post hoc power to detect differences between CS and GA was $51 \%$ for Tan $0-1$ versus $28.5 \%$ for Tan 2-3 regarding mRS 0-2 after 3 months (On-line Table 1).

\section{Association of Sedation Modes with Outcome Prediction by Radiologic Scores}

Correlation analysis for the entire cohort showed only an association between infarct volume and NIHSS change after 24 hours
Table 1: Baseline demographic and clinical characteristics according to no or poor (Tan 0-1) and moderate or good collaterals (Tan 2-3)

\begin{tabular}{|c|c|c|c|}
\hline & $\begin{array}{l}\operatorname{Tan} 0-1 \\
(n=48)\end{array}$ & $\begin{array}{l}\operatorname{Tan} 2-3 \\
(n=56)\end{array}$ & $\begin{array}{c}P \\
\text { Value }\end{array}$ \\
\hline \multicolumn{4}{|l|}{ Demographic characteristics } \\
\hline Age (mean) (SD) (yr) & $72.0(11.7)$ & $73.7(4.3)$ & $.52^{\mathrm{a}}$ \\
\hline Female sex (No.) (\%) & $17(35.4)$ & $23(41.1)$ & $.56^{\mathrm{b}}$ \\
\hline \multicolumn{4}{|l|}{ Premedication (No.) (\%) } \\
\hline Antiplatelets & & & $.5^{\mathrm{b}}$ \\
\hline No & $33(71.7)$ & $38(69.1)$ & \\
\hline Aspirin & $12(26.1)$ & $13(23.6)$ & \\
\hline Dual & $1(2.2)$ & $4(7.3)$ & \\
\hline Missing & 2 & 1 & \\
\hline \multicolumn{4}{|l|}{ Oral anticoagulants } \\
\hline No & $42(89.4)$ & $36(65.5)$ & $.01^{\mathrm{b}}$ \\
\hline Missing & 1 & 1 & \\
\hline \multicolumn{4}{|l|}{ Statins } \\
\hline No & $37(80.4)$ & $30(56.6)$ & $.01^{\mathrm{b}}$ \\
\hline Missing & 2 & 3 & \\
\hline \multicolumn{4}{|l|}{ Vascular risk factors (No.) (\%) } \\
\hline Hypertension & $37(77.1)$ & $37(66.1)$ & $.22^{\mathrm{b}}$ \\
\hline Diabetes mellitus & $13(27.1)$ & $10(17.9)$ & $.26^{\mathrm{b}}$ \\
\hline Hyperlipidemia & $12(25.0)$ & $17(30.4)$ & $.54^{\mathrm{b}}$ \\
\hline Smoking ${ }^{c}$ & $11(23.4)$ & $4(7.1)$ & $.02^{\mathrm{b}}$ \\
\hline Heart failure & $10(20.8)$ & $19(27.9)$ & $.14^{\mathrm{b}}$ \\
\hline Atrial fibrillation & $24(50)$ & $30(53.6)$ & $.72^{\mathrm{b}}$ \\
\hline Peripheral artery occlusive disease ${ }^{d}$ & $1(2.1)$ & $4(7.4)$ & $.22^{\mathrm{b}}$ \\
\hline \multicolumn{4}{|l|}{ Pretreatment imaging (No.) (\%) } \\
\hline ASPECTS & & & $<.001^{\mathrm{b}}$ \\
\hline $10-8$ & $24(50)$ & $47(83.9)$ & \\
\hline $7-6$ & $22(45.8)$ & $7(12.5)$ & \\
\hline$<6$ & $2(4.2)$ & $2(3.6)$ & \\
\hline Median (IQR) & $7(6-9)$ & $9(8-10)$ & \\
\hline Infarct volume (mean) (SD) $\left(\mathrm{cm}^{3}\right)$ & $107.4(99.1)$ & $35.0(55.1)$ & $<.001^{\mathrm{a}}$ \\
\hline \multicolumn{4}{|l|}{ Scores on admission (No.) (\%) } \\
\hline Premorbid mRS & & & $.02^{\mathrm{e}}$ \\
\hline 0 & $30(62.5)$ & $24(42.9)$ & \\
\hline 1 & $11(22.9)$ & $10(17.9)$ & \\
\hline 2 & $4(8.3)^{\prime}$ & $14(25)$ & \\
\hline$>2$ & $3(6.3)$ & $8(14.3)$ & \\
\hline NIHSS on admission & $17.9(3.6)$ & $16.9(3.7)$ & $.14^{\mathrm{a}}$ \\
\hline GCS on admission & & & $.8^{\mathrm{e}}$ \\
\hline 12 & $6(12.5)$ & $2(3.6)$ & \\
\hline 13 & $21(43.8)$ & $31(55.4)$ & \\
\hline $15-14$ & $21(43.8)$ & $23(41.2)$ & \\
\hline \multicolumn{4}{|l|}{ Occlusion (No.) (\%) } \\
\hline MCA & $26(54.2)$ & $39(69.6)$ & $.08^{\mathrm{b}}$ \\
\hline ICA & $3(6.3)^{\prime}$ & $0(0)$ & \\
\hline $\mathrm{ICA}+\mathrm{MCA}$ & $19(39.6)$ & $17(30.4)$ & \\
\hline Occlusion side right & $22(45.8)$ & $23(44.1)$ & $.63^{b}$ \\
\hline Treatment of stroke & & & $.24^{\mathrm{b}}$ \\
\hline IV tPA + EST & $32(66.7)$ & $31(55.4)$ & \\
\hline EST & $16(33.3)$ & $25(44.6)$ & \\
\hline
\end{tabular}

Note:-GCS indicates Glasgow Coma Scale; IQR, interquartile range.

${ }^{a} t$ test, 2-sided.

${ }^{\mathrm{b}} \chi^{2}$ test, 3-sided.

'Lower sample size in Tan group $0-1(n=47)$.

${ }^{d}$ Lower sample size in Tan group $0-1(n=47)$ and Tan group 2-3 $(n=54)$.

e Mann-Whitney $U$ test, 2-sided.

$(r=0.58)$ and between infarct volume and ASPECTS $(\rho=-0.6)$. An additional correlation analysis according to the applied sedation mode showed a moderate correlation between early NIHSS improvement and infarct volume $(r=0.71)$ and ASPECTS and infarct volume ( $\rho=-0.62)$ in the GA treatment group (On-line Table 2). Correlation analysis in the CS treatment group showed only a correlation between early NIHSS improvement and the mRS after 3 months $(\rho=0.61)$.

Regression analysis revealed that the presence of a moderate or good collateral status and favorable ASPECTS were strong predictors for smaller final mean infarct volume (coefficients: -51.75 ; 
Table 2: SIESTA primary and secondary outcome results according to no or poor (Tan 0-1) and moderate or good collaterals (Tan 2-3)

\begin{tabular}{|c|c|c|c|}
\hline & $\begin{array}{l}\text { Tan 0-1 } \\
(n=48)\end{array}$ & $\begin{array}{l}\text { Tan 2-3 } \\
(n=56)\end{array}$ & $P$ Value \\
\hline \multicolumn{4}{|l|}{ Primary outcome } \\
\hline Improvement in NIHSS (mean) (SD) & $-1.1(10.7)$ & $-5.8(7.6)$ & $.01^{\mathrm{a}}$ \\
\hline \multicolumn{4}{|l|}{ Secondary outcomes } \\
\hline \multicolumn{4}{|l|}{ Clinical (No.) (\%) } \\
\hline mRS 0-2 after 3 mo & $10(20.8)$ & $14(25.0)$ & $.82^{\mathrm{b}}$ \\
\hline In-house mortality & $7(14.6)$ & $1(1.8)$ & $.02^{\mathrm{b}}$ \\
\hline Mortality after $3 \mathrm{mo}$ & $12(25.0)$ & $15(26.8)$ & $.84^{\mathrm{b}}$ \\
\hline \multicolumn{4}{|l|}{ Logistics (mean) (SD) } \\
\hline Length of stay in hospital (days) & $6.0(3.7)$ & $4.6(2.6)$ & $.06^{\mathrm{c}}$ \\
\hline Length of stay in ICU (half-days) & $8.2(9.2)$ & $2.7(2.2)$ & $<.001^{\mathrm{c}}$ \\
\hline Length of ventilation (hr) & $30.1(5.9)$ & $3.8(5.4)$ & $.11^{\mathrm{C}}$ \\
\hline Length of stay on stroke unit (half-days) ${ }^{f}$ & $7.6(4.3)$ & $7.0(4.3)$ & $.5^{c}$ \\
\hline Onset-to-treatment (IV or IA) (min) ${ }^{g}$ & $122.8(64.8)$ & $129.6(60.9)$ & $.61^{\mathrm{b}}$ \\
\hline Door-to-groin (min) & $71.5(32.1)$ & $66.1(21.4)$ & $.52^{\mathrm{c}}$ \\
\hline Door-to-reperfusion (min) ${ }^{\mathrm{h}}$ & $174.8(57.1)$ & $165.6(60.5)$ & $.32^{c}$ \\
\hline \multicolumn{4}{|l|}{ Feasibility of EST (No.) (\%) } \\
\hline Degree of reperfusion (TICl) & & & $.97^{\mathrm{d}}$ \\
\hline $0-1$ & $3(6.3)$ & $5(9)$ & \\
\hline $2 a$ & $4(8.3)$ & $3(5.4)$ & \\
\hline $2 b$ & $24(50.0)$ & $25(44.6)$ & \\
\hline 3 & $17(35.4)$ & $23(41.1)$ & \\
\hline Substantial patient movement & $1(2.1)$ & $2(3.6)$ & $.65^{\mathrm{b}}$ \\
\hline Difficult vascular approach & $3(6.3)$ & $7(12.5)$ & $.28^{\mathrm{b}}$ \\
\hline Other & $3(6.3)$ & $8(14.3)$ & $.18^{\mathrm{b}}$ \\
\hline \multicolumn{4}{|l|}{ Safety (No.) (\%) } \\
\hline \multicolumn{4}{|l|}{ Complications before EST } \\
\hline Impaired monitor installation & $0(0.0)$ & $1(1.8)$ & $.35^{\mathrm{b}}$ \\
\hline Other & $1(2.1)$ & $0(0.0)$ & $.27^{\mathrm{b}}$ \\
\hline \multicolumn{4}{|l|}{ Complications during EST } \\
\hline Critical ventilation or oxygenation disturbance & $2(4.2)$ & $1(1.8)$ & $.47^{\mathrm{b}}$ \\
\hline Intervention-associated complications & $0(0.0)$ & $2(3.6)$ & $.19^{\mathrm{b}}$ \\
\hline $\begin{array}{l}\text { Vessel perforation with ICH and/or SAH complications } \\
\text { after EST }\end{array}$ & $0(0.0)$ & $2(3.6)$ & $.19^{\mathrm{b}}$ \\
\hline Hyper- or hypothermia & $12(25.0)$ & $12(21.4)$ & $.67^{b}$ \\
\hline Delayed extubation & $17(35.4)$ & $12(21.4)$ & $.11^{\mathrm{b}}$ \\
\hline Ventilation-associated complications & $6(12.5)$ & $3(5.4)$ & $.2^{\mathrm{b}}$ \\
\hline Ventilation-associated pneumonia & $6(12.5)$ & $3(5.4)$ & $.2^{\mathrm{b}}$ \\
\hline
\end{tabular}

Note:-IA indicates intra-arterial; min, minimum; max, maximum; ICH, intracerebral hemorrhage; ICU, intensive care unit.

${ }^{a}$ ANCOVA, NIHSS as baseline covariate.

${ }^{\mathrm{b}} \chi^{2}$ test, 2 -sided.

' $t$ test, 2 -sided.

${ }^{d}$ Mann-Whitney $U$ test, 2-sided.

e Lower sample size (41 and 46).

${ }^{\mathrm{f}}$ Lower sample size ( 31 and 51$)$.

${ }^{\circ}$ Lower sample size (41 and 47).

${ }^{\mathrm{h}}$ Lower sample size (45 and 49).

$95 \% \mathrm{CI},-81.58$ to $-21.92 ; P=.0008$; and $-2.36 ; 95 \% \mathrm{CI},-3.58$ to $-1.14 ; P<.0002$, respectively) (On-line Tables 3 and 4 ).

However, when we tested the interaction between the Tan group and sedation mode, no significant associations with any of the outcome measures were observed.

\section{DISCUSSION}

This post hoc analysis of the SIESTA trial showed a strong association of moderate-to-good collateral status with several surrogates of thrombectomy success in the entire cohort. Subdividing the cohort into 2 collateral status categories and those divided further according to the chosen sedation mode (CS or GA) did not show a relevant impact of either sedation mode on most associations.

The strong impact of collateral status is consistent with the results published in other recent studies. ${ }^{3,4,20}$ Our study adds to this evidence that the strong predictive value of the collateral score of Tan can be found even in a broad-practice RCT stroke population without rigid clinical or radiologic selection criteria. Although recent studies have suggested that collateral status is very variable in patients and that a good collateralization is strongly associated with smaller resulting infarcts and a better functional outcome, ${ }^{20,21}$ none of these studies reported data about the peri-interventional management or the sedation concept. Collateral status appears to be a very robust predictor not vulnerable to sedation mode with regard to most parameters.

We found, however, a few exceptions for patients treated with GA: In both collateral categories, these patients were more likely to experience delayed extubation than those started in CS and intubated during the intervention. Delayed extubation was often a consequence of spontaneous hypothermia after GA. Those with moderate or good collaterals, in particular, had a higher prevalence of postinterventional hypothermia and more delayed extubation. These patients also had a longer mean time-to-treatment (IV or intraarterial). This delay of treatment initiation in GA Tan 2-3 may have been caused by these patients having presented with fluctuating symptoms, which may have postponed the indication for EST.

Most interesting and relevant to our initial hypothesis, those with poor or no collaterals if treated under GA were more often independent (mRS $0-2$ ) at 3 months compared with the same collateral status group treated in CS, even though the results were only of marginal significance.

Our post hoc analysis is the first study to suggest that the predictive value of the Tan collateralization score and the ASPECTS on outcome does not appear to be influenced by the choice of peri-interventional CS or GA. Either such an influence does not exist or, more likely, SIESTA's protocols with strict target values for blood pressure and $\mathrm{CO}_{2}$ may have prevented compromising effects. As outlined above, an optimally performed GA may have its advantages compared with CS. The marginal significance for a favorable outcome after 3 months in the GA treatment arm of patients with poor collaterals may indicate such an advantage. Relative hypoventilation with slight hyper- 
carbia and cerebral vasodilation or at least stabilization of normocarbia may have improved the function of the few collaterals that remained. Alternative mechanisms may have been the potential neuroprotective effects of the applied sedatives ${ }^{22}$ or the significantly higher rate of hypothermia under GA. These speculations, however, demand further prospective mechanistically designed studies to assess how GA is best conducted during EST.

Our study has several limitations. First, SIESTA was a singlecenter study, and its findings are difficult to generalize; the same is true for post hoc analyses. Second, very small sample sizes of the subgroups limit insights from this explorative study. Third, in SIESTA, exclusively single-phase CTAs were conducted, which could have led to insufficient categorization of collateralization concerning temporal collaterals. Finally, interpretation of the CTAs may have been influenced by high-grade stenoses of the ICA or a pre-existent congestive heart failure for which we had not controlled.

\section{CONCLUSIONS}

Our results suggest that the benefit from thrombectomy can be expected in patients with moderate and, especially, good collaterals largely irrespective of the chosen sedation mode, if both general anesthesia and conscious sedation are performed according to protocols involving physiology parameter targets. It is still possible that a suboptimally performed GA during EST may eliminate the advantages of a good collateral status.

More prospective, multicenter studies with higher numbers of patients are necessary to clarify whether patients, particularly those with poor collateral statuses, may benefit from certain neuroprotective effects of GA or other sedation modes during thrombectomy.

Disclosures: Johannes Pfaff_UNRELATED: Payment for Lectures Including Service on Speakers Bureaus: Siemens; Travel/Accommodations/Meeting Expenses Unrelated to Activities Listed: Stryker. Simon Nagel—UNRELATED: Consultancy: Brainomix; Grants/Grants Pending: Brainomix*; Payment for Lectures Including Service on Speakers Bureaus: Medtronic, Pfizer, Bayer; Travel/Accommodations/Meeting Expenses Unrelated to Activities Listed: Bayer, Boehringer Ingelheim, Medtronic, Pfizer. Peter A. Ringleb_UNRELATED: Consultancy: Bayer, Boehringer Ingelheim, Covidien, Comments: Advisory Board member; Payment for Lectures Including Service on Speakers Bureaus: Bayer, Bristol-Myers Squibb, Boehringer Ingelheim, Daiichi Sankyo, Pfizer. Werner Hacke-UNRELATED: Consultancy: Boehringer Ingelheim, Medtronic, Neuravi; Grants/Grants Pending: Boehringer Ingelheim, Comments: grant for ECASS 4 study*; Payment for Lectures Including Service on Speakers Bureaus: Boehringer Ingelheim, Medtronic, Daiichi Sanyo, Neuravi. Martin BendszusUNRELATED: Board Membership: Data and Safety Monitoring Board for Vascular Dynamics, Guerbet, Boehringer Ingelheim; Consultancy: Codman, Hoffmann-La Roche, Guerbet, Boehringer Ingelheim; Grants/Grants Pending: Deutsche Forschungsgemeinschaft, Hopp Foundation, Novartis, Siemens, Guerbet, Stryker, Covidien*; Payment for Lectures Including Service on Speakers Bureaus: Novartis, Hoffmann-La Roche, Guerbet, Teva Pharmaceutical Industries, Bayer, Codman. Markus A. Möhlenbruch—UNRELATED: Payment for Lectures Including Service on Speakers Bureaus: Codman, Medtronic, MicroVention, Stryker, phenox. Julian BöselUNRELATED: Grants/Grants Pending: Patient-Centered Outcomes Research Institute, Comments: award SETPOINT2 study*; Payment for Lectures Including Service on Speakers Bureaus: C.R. BARD, ZOLL, Seiratherm, Boehringer Ingelheim, Sedana Medical, Comments: speaker honoraria and travel support; Travel/Accommodations/Meeting Expenses Unrelated to Activities Listed: C.R. BARD, ZOLL, Seiratherm, Boehringer Ingelheim, Sedana Medical. *Money paid to the institution.

\section{REFERENCES}

1. Goyal M, Menon BK, van Zwam WH, et al; HERMES collaborators. Endovascular thrombectomy after large-vessel ischaemic stroke: a meta-analysis of individual patient data from five randomised trials. Lancet 2016;387:1723-31 CrossRef Medline

2. Saver JL, Goyal M, van der Lugt A, et al; HERMES Collaborators. Time to treatment with endovascular thrombectomy and outcomes from ischemic stroke: a meta-analysis. JAMA 2016;316:1279-88 CrossRef Medline

3. Bang OY, Saver JL, Kim SJ, et al. Collateral flow predicts response to endovascular therapy for acute ischemic stroke. Stroke 2011;42: 693-99 CrossRef Medline

4. Liebeskind DS. Collateral circulation. Stroke 2003;34:2279-84

5. Liebeskind DS, Tomsick TA, Foster LD, et al; IMS III Investigators. Collaterals at angiography and outcomes in the Interventional Management of Stroke (IMS) III trial. Stroke 2014;45:759-64 CrossRef Medline

6. Berkhemer OA, Jansen IG, Beumer D, et al; MR CLEAN Investigators. Collateral status on baseline computed tomographic angiography and intra-arterial treatment effect in patients with proximal anterior circulation stroke. Stroke 2016;47:768-76 CrossRef Medline

7. Reich DL, Hossain S, Krol M, et al. Predictors of hypotension after induction of general anesthesia. Anesth Analg 2005;101: 622-28 Medline

8. Mundiyanapurath S, Stehr A, Wolf M, et al. Pulmonary and circulatory parameter guided anesthesia in patients with ischemic stroke undergoing endovascular recanalization. J Neurointerv Surg 2016;8: 335-41 CrossRef Medline

9. Lowhagen Hendén P, Rentzos A, Karlsson JE, et al. Hypotension during endovascular treatment of ischemic stroke is a risk factor for poor neurological outcome. Stroke 2015;46:2678-80 CrossRef Medline

10. Davis MJ, Menon BK, Baghirzada LB, et al; Calgary Stroke Program. Anesthetic management and outcome in patients during endovascular therapy for acute stroke. Anesthesiology 2012;116:396-405 CrossRef Medline

11. Takahashi CE, Brambrink AM, Aziz MF, et al. Association of intraprocedural blood pressure and end tidal carbon dioxide with outcome after acute stroke intervention. Neurocrit Care 2014;20: 202-08 CrossRef Medline

12. Brinjikji W, Murad MH, Rabinstein AA, et al. Conscious sedation versus general anesthesia during endovascular acute ischemic stroke treatment: a systematic review and meta-analysis. AJNR AmJ Neuroradiol 2015;36:525-29 CrossRef Medline

13. Schönenberger S, Möhlenbruch M, Pfaff J, et al. Sedation vs. Intubation for Endovascular Stroke TreAtment (SIESTA): a randomized monocentric trial. Int J Stroke 2015;10:969-78 CrossRef Medline

14. Schönenberger S, Uhlmann L, Hacke W, et al. Effect of conscious sedation vs general anesthesia on early neurological improvement among patients with ischemic stroke undergoing endovascular thrombectomy: a randomized clinical trial. JAMA 2016;316: 1986-96 CrossRef Medline

15. Menon BK, Puetz V, Kochar P, et al. ASPECTS and other neuroimaging scores in the triage and prediction of outcome in acute stroke patients. Neuroimaging Clin N Am 2011;21:407-23, xii CrossRef Medline

16. Tan IY, Demchuk AM, Hopyan J, et al. CT angiography clot burden score and collateral score: correlation with clinical and radiologic outcomes in acute middle cerebral artery infarct. AJNR Am J Neuroradiol 2009;30:525-31 Medline

17. Tomsick T, Broderick J, Carrozella J, et al; Interventional Management of Stroke II Investigators. Revascularization results in the Interventional Management of Stroke II trial. AJNR Am J Neuroradiol 2008;29:582-87 Medline

18. Zaidat OO, Yoo AJ, Khatri P, et al; Cerebral Angiographic Revascularization Grading (CARG) Collaborators, STIR Revascularization working group, STIR Thrombolysis in Cerebral Infarction (TICI) Task Force. Recommendations on angiographic revascularization grading standards for acute ischemic stroke: a consensus statement. Stroke 2013;44:2650-63 CrossRef Medline

19. The R Core Team. A Language and Environment for Statistical Com- 
puting. R Vienna: Foundation for Statistical Computing, Vienna, Austria; 2016

20. Liebeskind DS. Collateral lessons from recent acute ischemic stroke trials. Neurol Res 2014;36:397-402 CrossRef Medline

21. Menon BK, Smith EE, Modi J, et al. Regional leptomeningeal score on CT angiography predicts clinical and imaging outcomes in pa- tients with acute anterior circulation occlusions. AJNR Am J Neuroradiol 2011;32:1640-45 CrossRef Medline

22. Sivasankar C, Stiefel M, Miano TA, et al. Anesthetic variation and potential impact of anesthetics used during endovascular management of acute ischemic stroke. J Neurointerv Surg 2016;8:1101-06 CrossRef Medline 